\title{
AUTOEVALUACIÓN DE LA COMPETENCIA INFORMACIONAL EN LOS ESTUDIOS DE PSICOLOGÍA DESDE LA PERCEPCIÓN DEL ESTUDIANTE
}

\author{
María Pinto Molina* \\ Facultad de Comunicación y Documentación. Universidad de Granada. \\ Susana Puertas Valdeiglesias **
}

Facultad de Psicología. Universidad de Granada.

\begin{abstract}
Resumen: Teniendo como referencia la implantación del Espacio Europeo de Educación Superior, se aborda la autoevaluación de las competencias informacionales por parte de los estudiantes universitarios de Psicología. Se utiliza como instrumento de diagnóstico el cuestionario IL-HUMASS, que centra su atención en estas cuatro dimensiones clave: búsqueda, evaluación, procesamiento y comunicación-difusión de la información y desde un triple enfoque: importancia, autoeficacia y hábitos de aprendizaje. Se presentan los resultados obtenidos destacando cuáles son las competencias que mejor y peor valoran los estudiantes según los cursos y las que consideran que tienen mayor competencia, así como cuales son las que más dificultades tienen en su comprensión y puesta en práctica. Como conclusiones se ofrece una propuesta de intervención que ayude a mejorar las debilidades detectadas en su competencia informacional.
\end{abstract}

Palabras clave: alfabetización informacional; competencia informacional; psicología; búsqueda de información; evaluación de la información; procesamiento de la información; comunicación de la información; cuestionario IL-HUMASS.

Title: INFORMATION COMPETENCE SELF-ASSESSMENT IN PSYCHOLOGY STUDIES FROM STUDENTS' PERCEPTION.

Abstract: Taking into account the establishment of the European Higher Education Area, an approach into the self-assessment of information competences by students of Psychology is proposed. Our diagnostic tool is the IL-HUMASS survey, focused on four key dimensions: information search, evaluation, processing and communication-diffusion. The questionnaire also has a three-faceted viewpoint: motivation, self-efficacy and learning habits from the students. We present the results enhancing which are the competences best and worst evaluated by students, in diverse courses; which ones they consider they manage better, which ones they consider as more important; and which seem more difficult for them, regarding understanding and development. In our conclusions we will offer a practical proposal to intervene and help the students in order to improve the weaknesses diagnoses in their information competence.

\footnotetext{
*mpinto@ugr.es

**spuerta@ugr.es
}

Recibido: 25/04/2012; aceptado: 01/06/2012.

PINTO MOLINA, M. y PUERTAS VALDEIGLESIAS, S. Autoevaluación de la competencia informacional en los estudios de Psicología desde la percepción del estudiante. Anales de Documentación, 2012, vol. 15, nº 2. ISSN: 1697-7904. http://dx.doi.org/10.6018/analesdoc.15.2.151661. 
Keywords: information literacy; information competencies; psychology; information search; evaluation of the information; information processing; communication of information; IL-HUMASS survey.

\section{INTRODUCCIÓN}

Fundamentalmente, el ejercicio de la Psicología se dirige hacia la mejora de la calidad de vida humana y social; contempla entre sus principales objetivos el bienestar, la salud y el pleno desarrollo de los individuos, los grupos, las organizaciones y, en definitiva, de la sociedad en sus diversos ámbitos. Los psicólogos trabajan estrechamente con profesionales de otros ámbitos, en grupos multidisciplinares, y su contribución particular es el resultado del entrenamiento y el conocimiento acerca de los aspectos psicológicos de los problemas y situaciones, desde una perspectiva y método científicos.

Del mismo modo, la Psicología como disciplina científica se centra en la descripción, comprensión, predicción y control de las manifestaciones conductuales de los procesos psicológicos de tipo cognitivo, motivacional y/o actitudinal en una amplia variedad de contextos (sanitario, educativo, laboral, social...), dicho de otro modo, es el estudio científico del comportamiento y de los procesos mentales (Papalia y Wendkos, 1993). En este sentido, el licenciado en psicología deberá disponer de la formación científica y de las capacidades técnicas necesarias para la resolución de problemas psicológicos socialmente relevantes que puedan tener lugar en dichos contextos. Para ello será indispensable que adquieran competencia en alfabetización informacional, una competencia básica que les permitirá acceder, tratar, evaluar, elaborar y comunicar la información pertinente en su área de una manera óptima y correcta a partir de determinadas fuentes de información.

En España, la licenciatura en Psicología ha sido desde hace tiempo una carrera de dos ciclos distribuidos en cinco años, pero tras la adaptación al Espacio Europeo de Educación Superior (EEES) se ha transformado en un Título de Grado de cuatro años, que trata de adaptarse a los postulados del EEES mediante la redefinición de la carga de trabajo de los estudiantes a través de los créditos ECTS (European Credits Transfer System) y una reorganización conceptual de los sistemas educativos. Ello supone pasar de una actividad docente, centrada en el profesor y definida por el hecho de "enseñar a aprender", a un proceso de aprendizaje activo del estudiante, basado en "aprender a aprender". El profesor deberá formar un estudiantado autónomo capaz de aprender por sí mismo y por lo tanto de autorregular su actividad profesional de manera flexible, en función de las demandas sociales y de mercado, a partir de una formación integral por competencias (OCDE, 2005). Siguiendo las recomendaciones de los proyectos Tuning (González, Wagenaar, 2003), Rychen y Salganik, (2003) y Alfin-EEES (Pinto, 2005) el estudiante debe adquirir una serie de competencias genéricas comunes a cualquier titulación, relacionadas con las nuevas tecnologías, la habilidad para la comunicación oral y escrita, la capacidad de análisis y síntesis, espíritu crítico, habilidad para el trabajo individual y en grupo, integración en equipos multidisciplinares, preocupación por la calidad, capacidad de liderazgo, etc., que van a ser herramientas básicas en su vida profesional. 
Por consiguiente, el objetivo principal de este artículo es ofrecer un diagnóstico de la percepción que tienen los estudiantes de Psicología sobre su conocimiento y dominio de la competencia informacional, atendiendo a estas cuatro macrocompetencias clave: búsqueda de información, evaluación, procesamiento y comunicación-difusión de la información. Pensamos que esta especie de radiografía desde la perspectiva del estudiante ayudará a orientar las iniciativas de evaluación adecuadas que mejor midan el logro de estas competencias y conduzcan a propuestas de intervención educativa efectivas.

\section{REVISIÓN DE LA LITERATURA}

Hoy por hoy el estudiante no necesita sólo saber utilizar la biblioteca, sino poseer habilidades y estrategias para informarse y usar la información para la toma de decisiones en su proceso formativo y de aprendizaje.

A diferencia de la formación de usuarios, la alfabetización informacional (ALFIN) enfoca la formación de usuarios desde una perspectiva de habilidades informacionales, basada en el uso crítico y estratégico de la información más allá de los servicios de la biblioteca. En la década de los años ochenta con el desarrollo e implantación de las tecnologías de información y comunicación (TIC) en el marco de las bibliotecas, el concepto de ALFIN se ve condicionado por este escenario, y algunos autores como Breivik (1989) y Kuhlthau (1999) lo asocian a la alfabetización tecnológica y al aprendizaje centrado en recursos. En 1989 el Comité Presidencial de ALA publica un informe final de amplio espectro dirigido a ciudadanos, trabajadores, estudiantes, profesores, bibliotecarios..., apoyando la implantación de un nuevo modelo de aprendizaje, más dinámico y enfocado al aprendizaje de habilidades para el manejo y uso crítico de la información. En esta línea se han pronunciado Doyle (1994), Beherens (1994), Bruce $(1996,1997)$ o Huston (1999) entre otros, quienes afirman que ALFIN es un conjunto de aptitudes para localizar, manejar, evaluar y utilizar la información de forma eficaz para una gran variedad de propósitos.

En el ámbito de la Psicología despuntan una serie de estudios sobre la competencia informacional de los estudiantes, aunque podemos decir que no abunda mucha información específica sobre el tema. Algunos trabajos, como los de Sutton (1995), abordan aspectos de la alfabetización informacional relacionados con el uso de los recursos, y afirman que los estudiantes que utilizan como instrumentos para el aprendizaje, entre otros, presentaciones de conferencias o tutoriales, son competentes en el uso de los recursos electrónicos, especialmente en la consulta de bases de datos bibliográficas (como PsycLIT / PsycINFO) y en el manejo de Internet. Otros estudios como los de Daugherty (1997) y Carter (1998), analizan los pasos y la metodología que los estudiantes emplean para obtener un artículo de revista sobre un tema específico de psicología. Además, proponen sesiones de formación para mejorar las estrategias de búsqueda orientadas a la investigación y como instrumento de evaluación utilizan un pre y postest para medir los resultados. Thaxton (2002) destaca la importancia que tiene en los estudios de Psicología las competencias informacionales. En un estudio exploratorio, 
entrevista a cinco prestigiosos psicólogos de la Universidad Estatal de Georgia sobre estos tres aspectos: (1) relevancia actual del modelo de comunicación Garvey-Griffith (referencia); (2) impacto de los cambios ocurridos en las tecnologías de la información y comunicación; y (3) percepciones de los estudiantes sobre sus necesidades de información y el papel formador de la biblioteca. La mayoría de los participantes resaltaron la prevalencia del modelo de difusión de Garvey-Griffith en casi todas las subdisciplinas de la Psicología, aunque sin perder de vista el valor de Internet para la comunicación profesional y académica, que ha llevado a un modelo más circular.

Otro trabajo de interés es el de Larkin y Pines (2005), quienes, partiendo de un estudio de casos, plantean la importancia que para muchos profesores de Psicología tienen las competencias informacionales en el currícula formativo de los estudiantes. Otras iniciativas abordan la integración de la alfabetización informacional en los planes de estudios. Un ejemplo claro es el de la Universidad de Georgia State (GSU), que considera ALFIN como una necesidad para la formación de los estudiantes de esta especialidad. Thaxton (2002) y Thaxton, Faccioli y Page (2004) hablan de la realización de cursos específicos, centrados en aspectos relacionados con la búsqueda, organización, evaluación y difusión de la información en Psicología. Paglia y Donahue (2003), aluden a la integración de las competencias informacionales en los planes de estudio de Psicología en la Universidad de New Hampshire. En esta línea de colaboración entre académicos y bibliotecarios se enmarcan las acciones de alfabetización informacional en el área de Psicología de la Facultad de Educación de la California State University Northridge. La experiencia de esta universidad en la implementación de programas de alfabetización informacional, comenzó en la década de 1990 con la creación del Information Competence Work Group, compuesto por bibliotecarios y docentes, con el fin de desarrollar iniciativas para sensibilizar sobre la necesidad de formación de habilidades de información en todos los campus de la propia universidad (Lampert, 2005). Por otra parte, en la Bridgewater State College se implementó en el año 2006 el curso "The Psychology of Academic Success" con el propósito de potenciar habilidades de información en los estudiantes de psicología (Hayes-Bohanan y Spievak, 2008). En esta línea ya la Universidad de Griffith planteó en 1999 una iniciativa para el desarrollo de habilidades de información conocida como Griffith Graduate Project (Abbott y Peach, 2000). Otros estudios comparan las expectativas e intenciones de los bibliotecarios con las percepciones y conductas de los estudiantes de grado respecto al uso de los recursos de información (Sadler y Given, 2007).

En España, ya están en funcionamiento los nuevos Grados adaptados a la filosofía del Espacio Europeo de Educación Superior (ANECA, 2004; MICINN, 2007), en los que destaca el protagonismo de la formación por competencias, especialmente aquellas transversales que son clave para el proceso formativo de los estudiantes. De éstas, en este estudio abordamos la competencia informacional, basada en la capacidad del estudiante de reunir e interpretar datos relevantes para emitir juicios de valor que incluyan una reflexión crítica sobre temas relevantes de índole científica, social..., así como la capacidad para 
gestionar, recuperar, analizar, sintetizar, transmitir y utilizar la información de forma ética y legal.

En consonancia con lo que señalan estudios recientes (Gross y Latham, 2009), se sabe poco acerca de cómo los estudiantes conceptualizan sus experiencias y comportamientos relacionados con la información. Por eso, el empleo de herramientas de autoevaluación para conocer la percepción que tienen los estudiantes sobre sus habilidades informacionales es una práctica frecuente en la revisión de la literatura (Colthart et al., 2008). Entre otros muchos, un ejemplo de autoevaluación es la investigación realizada por Julien y Baker (2009) basada en la triangulación de datos procedentes de dos métodos de trabajo, por un lado la resolución por parte de los estudiantes de actividades realizadas en el aula centradas en la búsqueda de información y por otro, la información procedente de entrevistas semiestructuradas.

Por tanto, consideramos que será de utilidad conocer de primera mano qué piensan los estudiantes de Psicología sobre su nivel de autoeficacia en relación con las principales competencias informacionales y qué importancia le conceden en el marco de su formación. Ello nos permitirá conocer qué piensan los estudiantes sobre su pericia en el manejo de la competencia informacional y además, nos marcará pautas e indicadores para orientar las propuestas de evaluación adecuadas que mejor midan la adquisición de estas competencias y diseñar programas de intervención educativa adaptados a las necesidades reales del estudiantado y que, por tanto, resultan más eficientes. Una vez más el rol del profesor-tutor es clave, pues además de transmitir conocimientos específicos, debe sensibilizar y orientar al estudiante en cómo usar la información para afrontar sus necesidades de aprendizaje (Maybee, 2006). Pero profesores y bibliotecarios han de pensar en términos de resultados del aprendizaje del estudiante (Hernon, 2006), es decir, qué debe saber hacer el estudiante después de haber completado su proceso de aprendizaje. La American Psychological Association (APA) ha elaborado un documento guía sobre los resultados de aprendizaje asociados al título de grado en Psicología que puede resultar útil como una primera aproximación. Además, los resultados de aprendizaje deben ir asociados a criterios de evaluación eficaces, que tengan en cuenta tanto el aprendizaje de conocimientos formales (declarativos) como la formación en competencias y habilidades (procedimentales) genéricas y específicas que definen el catálogo formativo. De hecho, las experiencias de los últimos años van en esa línea. Encontramos, por ejemplo, el trabajo de McKinney, Jones y Turkington (2011) donde llevan a cabo la evaluación del currículum de los estudiantes del departamento de Psicología en la Universidad de Sheffield. Los estudiantes trabajaban de forma colaborativa en una tarea que consistía en generar historietas nuevas basadas en investigaciones psicológicas reales e investigar artículos científicos relacionados con dicha investigación. De igual manera, en la tarea tenían que encontrar las diferencias existentes entre ambas fuentes de información. La evaluación de la percepción del estudiante sobre su eficacia en la tarea se medía a través de diferentes instrumentos (cuestionario, test de competencia informacional, grupos focales y trabajo del estudiante). Los resultados demostraron que aunque los estudiantes desarrollaron su conocimiento y 
capacidad para buscar fuentes académicas apropiadas, preferían hacer búsquedas en Google Scholar que en la Web of Knowledge.

\section{MATERIAL Y MÉTODOS}

Para intentar dar respuesta a nuestros objetivos diseñamos este estudio en el que hemos utilizado como instrumento básico de recogida de información el cuestionario ALFIN-HUMASS que está desarrollado para ser administrado on-line.

El diseño del cuestionario se apoya en un amplio corpus de literatura dentro del campo de la alfabetización informacional, tanto de carácter general y normativo (Corrall, 2007; Webber, 2006; ACRL, 2000; SCONUL, 1999; Bruce, 1997; Bloom, 1956), como específico, desde la perspectiva del usuario y desde un enfoque evaluativo (Limberg, 2006; Maybe, 2006; Tuominen, Savolainen, Talja, 2005). El objetivo del cuestionario es proporcionar un autodiagnóstico de la competencia informacional en el ámbito de la enseñanza superior recabando la opinión de profesores, bibliotecarios y estudiantes, con el fin de conocer qué competencias son útiles para el proceso de enseñanza-aprendizaje, con el fin de incluir en los currícula programas y contenidos ALFIN que contribuyan a una formación estratégica y por competencias (Pinto, 2011). Se estructura en estas cuatro categorías: búsqueda de información; evaluación de la información; procesamiento; y comunicación y difusión de la información, y consta de 26 items, de los cuales once están relacionados con competencias digitales, lo que confirma la pertinencia de los temas tecnológicos en la alfabetización Informacional. Los ítems se conciben para que sean autoinformados por la población encuestada desde una perspectiva actitudinal, teniendo en cuenta estos tres factores: importancia, autoeficacia y hábitos de aprendizaje (Pinto, 2010).

Este cuestionario ha sido respondido por los estudiantes de la titulación de Psicología de las Universidades de Granada y Salamanca durante el año 2010.

La muestra estuvo compuesta por 501 personas donde 93 fueron hombres y 408 fueron mujeres. La media de edad fue de 21,7 años estando el intervalo de edades comprendido entre los 18 y 48 años de edad. El 36,7\% de la muestra cursaba primer año de la licenciatura de Psicología, el 21,2\% cursaba segundo año, el 12,6\% tercero, el 10,4\% cuarto, el $9 \%$ quinto, el 3,6\% cursaba estudios de máster y el 5,2\% cursaba estudios de doctorado.

La recogida de datos se realizó para toda la muestra en un laboratorio de informática perteneciente a la Facultad de Psicología bien de la Universidad de Granada o bien de la Universidad de Salamanca. El protocolo de instrucciones fue aplicado por una misma investigadora en cada una de las universidades para eliminar la posibilidad de sesgos en las respuestas. El anonimato de las respuestas estuvo garantizado y se les ofrecía al estudiantado la posibilidad de indicar voluntariamente su e-mail si querían conocer posteriormente los resultados del estudio. 
El cuestionario utilizado recoge información sociodemográfica y sobre todo obtiene datos referidos a cuatro grandes competencias transversales que son:

- Búsqueda de información: con 8 ítems diferentes

- Evaluación de la información: con 5 ítems diferentes

- Procesamiento de la información: con 6 ítems diferentes

- Comunicación de la información y la difusión: con 7 ítems diferentes

El estudiante tenía que responder para cada uno de los ítems señalados en tres escalas diferentes:

- Importancia: en una escala tipo Likert de 9 puntos donde 1 era baja y 9 era alta, el estudiante señalaba la importancia que le daba a cada competencia recogida en cada uno de los ítems de las 4 categorías señaladas anteriormente

- Autoeficacia: en una escala tipo Likert de 9 puntos donde 1 era baja y 9 era alta, el estudiante señalaba el nivel que consideraba que tenía para cada competencia recogida en cada uno de los ítems de las 4 categorías señaladas anteriormente

- Hábitos de aprendizaje: para cada ítem, el estudiante señalaba si esa competencia la había adquirido en clase, en cursos, en bibliotecas o de forma individual y autodidacta. Sólo se le permitía señalar una opción de las cuatro posibles

\section{RESULTADOS Y DISCUSIÓN}

En primer lugar realizamos un análisis de fiabilidad del instrumento que nos permitiera estar seguras de que los datos recogidos eran precisos y tenían sentido. Para ello agrupamos los ítems de cada categoría y para cada uno de los dos primeros niveles de manera que teníamos 8 factores principales: importancia de la búsqueda de información, nivel de la competencia de búsqueda, importancia de la evaluación de la información, nivel de la evaluación de la información, importancia del procesamiento de la información, importancia de la comunicación de la información y la difusión, y nivel adquirido de esta competencia. Los resultados del análisis de fiabilidad se muestran en la Tabla I.

\begin{tabular}{|l|l|}
\hline FACTOR & ALFA DE CRONBACH \\
\hline Importancia búsqueda & 0.82 \\
\hline Autoeficacia búsqueda & 0.85 \\
\hline Importancia evaluación & 0.78 \\
\hline Autoeficacia evaluación & 0.81 \\
\hline Importancia procesamiento & 0.79 \\
\hline Autoeficacia procesamiento & 0.73 \\
\hline Importancia comunicación & 0.82 \\
\hline Autoeficacia comunicación & 0.78 \\
\hline
\end{tabular}

Tabla I. Valores alfa de fiabilidad para los factores principales del instrumento. 
A la luz de los datos podemos afirmar que el instrumento ha resultado muy fiable ya que los índices para cada factor son altos y, por tanto es pertinente agrupar los ítems para realizar los análisis estadísticos posteriores.

Posteriormente realizamos un análisis de correlaciones entre los factores para explorar el funcionamiento del instrumento y los resultados fueron de altas correlaciones entre los factores, siendo todas ellas significativas tal y como se muestra a continuación en la Tabla II.

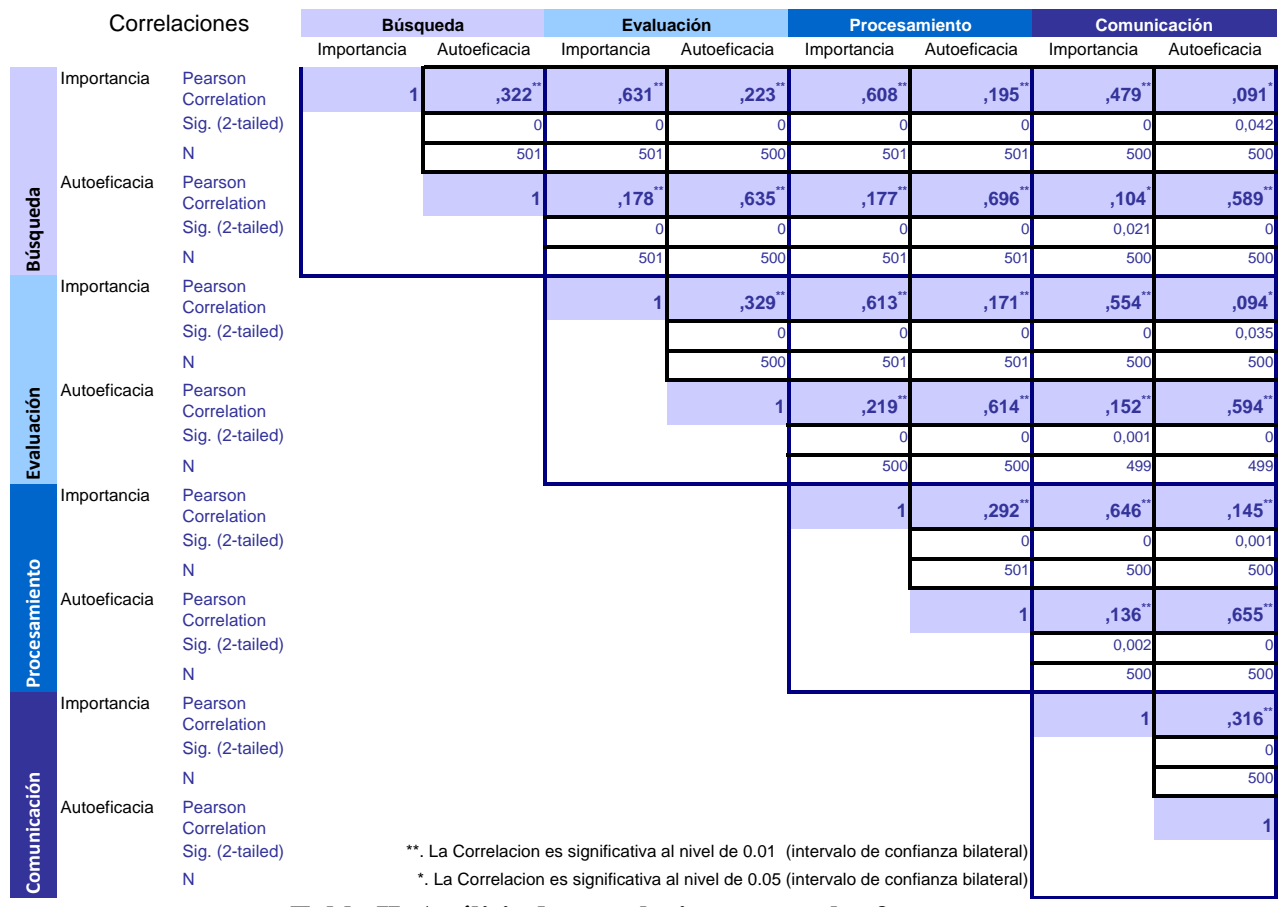

Tabla II. Análisis de correlaciones entre los factores.

Los resultados del análisis de correlaciones nos muestra un instrumento con alta consistencia interna, muy cohesionado y fiable para la recogida de datos. Además arroja un dato que, en nuestra opinión, es muy interesante ya que sugiere que los participantes en el estudio, cuanto más nivel creen que tienen adquirido de una competencia mayor importancia le dan a esa competencia. Nos parece un resultado importante, sobre todo, con vistas a la intervención, de manera que probablemente cuanto más se entrene al estudiantado en una competencia determinada y mayor nivel vayan adquiriendo en ella, mayor será la importancia y el valor que le den a la misma. 
Realizamos un análisis de medidas repetidas para los factores en la importancia de la competencia. Los resultados muestran diferencias significativas entre los factores, $\mathrm{F}(3,38)$ $=19,06$ y $\mathrm{p}<.000$. Las medias de cada factor se muestran en la Tabla III.

\begin{tabular}{|l|l|l|l|}
\hline & Media & Desv. típ. & N \\
\hline Búsqueda importancia & 7,60 &, 9 & 500 \\
Evaluación importancia & 7,87 &, 91 & 500 \\
Procesamiento importancia & 7,47 & 1,03 & 500 \\
Comunicación importancia & 7,94 &, 89 & 500 \\
\hline
\end{tabular}

Tabla III. Medias de los factores en cuanto a la importancia de la competencia.

Los resultados revelan que el factor más importante de competencias para el estudiantado fue el de comunicación y difusión de la información y al que menos importancia daban era al de procesamiento de la información.

Igualmente realizamos un análisis de medidas repetidas para los factores en el nivel de adquisición de la competencia. Los resultados muestran diferencias significativas entre los factores, $\mathrm{F}(3,38)=40,54 \mathrm{y} \mathrm{p}<.000$. Las medias de cada factor se muestran en la Tabla IV.

\begin{tabular}{|l|l|l|l|}
\hline & Media & Desv. típ. & N \\
\hline Búsqueda nivel & 6,2 & 1,26 & 499 \\
Evaluación nivel & 6,29 & 1,27 & 499 \\
Procesamiento nivel & 5,86 & 1,3 & 499 \\
Comunicación nivel & 6,14 & 1,28 & 499 \\
\hline
\end{tabular}

Tabla IV. Medias de los factores en cuanto al nivel adquirido de la competencia.

Los resultados indican que el factor más importante en el nivel de adquisición de competencias para el estudiantado fue el de evaluación de la información (en el que más formados se perciben) y el factor en el que menor nivel de adquisición creen que tienen era el de procesamiento de la información, precisamente el mismo factor al que menos importancia conceden. Parece pues, que el factor que creen menos importante es el que menos les preocupa en el sentido de que es al que menos interés prestan a la hora de formarse o mejorar en su nivel de competencia.

Realizando un análisis exploratorio general de los datos, encontramos que la competencia que consideran menos importante es la de "saber utilizar fuentes electrónicas informales de información" (media= 6,7) que está dentro del factor de búsqueda de información. Por el contrario, la competencia que consideran más importante de todas es la de "saber redactar un documento" (media= 8,5) que está dentro del factor de comunicación y difusión de la información. En cuanto al nivel de adquisición de las competencias, consideran que el mayor nivel adquirido lo tienen en la competencia "saber 
resumir y esquematizar la información" (media=7,5) dentro del factor de tratamiento de la información, y la competencia que consideran peor adquirida es la de "saber utilizar gestores de referencias bibliográficas" (media $=4,1$ ) precisamente dentro del mismo factor.

Si tenemos en cuenta el análisis por factores encontramos los resultados recogidos en la Tabla V:

\begin{tabular}{|c|c|c|c|c|}
\hline FACTOR & $\begin{array}{l}\text { COMPETENCIA } \\
\text { MÁS } \\
\text { IMPORTANTE }\end{array}$ & $\begin{array}{l}\text { COMPETENCIA } \\
\text { MENOS } \\
\text { IMPORTANTE }\end{array}$ & $\begin{array}{l}\text { COMPETENCIA } \\
\text { MEJOR } \\
\text { ADQUIRIDA }\end{array}$ & $\begin{array}{l}\text { COMPETENCIA } \\
\text { PEOR } \\
\text { ADQUIRIDA }\end{array}$ \\
\hline $\begin{array}{ll}\text { Búsqueda } & \text { de } \\
\text { información }\end{array}$ & $\begin{array}{ll}\text { Conocer } & \text { la } \\
\text { terminología } & \text { de } \\
\text { tu materia } & \end{array}$ & $\begin{array}{l}\text { Saber utilizar } \\
\text { fuentes } \\
\text { electrónicas } \\
\text { informales de } \\
\text { información }\end{array}$ & $\begin{array}{l}\text { Saber buscar y } \\
\text { recuperar } \\
\text { información en } \\
\text { internet }\end{array}$ & $\begin{array}{lr}\text { Conocer } & \text { las } \\
\text { estrategias } & \text { de } \\
\text { búsqueda } & \text { de } \\
\text { información } & \end{array}$ \\
\hline Medias & 8,4 & 6,7 & 7,2 & 4,8 \\
\hline $\begin{array}{l}\text { Evaluación de la } \\
\text { información }\end{array}$ & $\begin{array}{l}\text { Reconocer en el } \\
\text { texto las ideas } \\
\text { del autor }\end{array}$ & $\begin{array}{lr}\text { Conocer } & \text { la } \\
\text { tipología de las } \\
\text { fuentes } & \text { de } \\
\text { información } & \\
\text { científica } & \\
\end{array}$ & $\begin{array}{l}\text { Reconocer en el } \\
\text { texto las ideas } \\
\text { del autor }\end{array}$ & $\begin{array}{lr}\text { Conocer } & \text { la } \\
\text { tipología de las } \\
\text { fuentes } & \text { de } \\
\text { información } & \\
\text { científica } & \\
\end{array}$ \\
\hline Medias & 8,3 & 7,1 & 7,2 & 5,6 \\
\hline $\begin{array}{l}\text { Tratamiento de } \\
\text { información }\end{array}$ & $\begin{array}{l}\text { Saber resumir y } \\
\text { esquematizar la } \\
\text { información }\end{array}$ & $\begin{array}{l}\text { Saber instalar } \\
\text { programas } \\
\text { informáticos }\end{array}$ & $\begin{array}{l}\text { Saber resumir y } \\
\text { esquematizar la } \\
\text { información }\end{array}$ & $\begin{array}{l}\text { Saber utilizar } \\
\text { gestores de } \\
\text { referencias } \\
\text { bibliográficas }\end{array}$ \\
\hline Medias & 8,4 & 6,7 & 7,5 & 4,1 \\
\hline $\begin{array}{l}\text { Comunicación y } \\
\text { difusión de la } \\
\text { información }\end{array}$ & $\begin{array}{l}\text { Saber redactar } \\
\text { un documento }\end{array}$ & $\begin{array}{l}\text { Conocer } \\
\text { legislación sobre } \\
\text { el uso de la } \\
\text { información y de } \\
\text { la propiedad } \\
\text { intelectual }\end{array}$ & $\begin{array}{l}\text { Saber hacer } \\
\text { presentaciones } \\
\text { académicas }\end{array}$ & $\begin{array}{l}\text { Saber } \\
\text { comunicar en } \\
\text { otros idiomas }\end{array}$ \\
\hline Medias & 8,5 & 7,1 & 7,2 & 4,6 \\
\hline
\end{tabular}

Tabla V. Análisis competencial exploratorio de los datos por factores.

Nótese que tanto en el factor de evaluación de la información como en el de tratamiento de la información la competencia que consideran más importante es a su vez la competencia en la que se perciben mejor preparados y lo mismo ocurre con la competencia menos importante y en la que menos formados están en el factor de evaluación de la información.

A continuación, realizamos un análisis exploratorio por cursos para averiguar si había diferencias significativas en cuanto a importancia de la competencia y nivel adquirido de la misma en función del curso académico en el que estuvieran los participantes. Para este propósito se unieron los cursos de doctorado y máster por entender que tienen niveles similares y así tener una muestra más representativa, de esta forma teníamos estudiantes 
de primer, segundo, tercer, cuarto, quinto y sexto curso, entendiéndose por sexto los que cursaban doctorado o máster. Se realizó un ANOVA de un factor para averiguar nuestro propósito. En este análisis 5 de los 8 factores tienen diferencias significativas en función del curso que están realizando (importancia de la búsqueda, nivel de la búsqueda, importancia de la evaluación, nivel de evaluación e importancia del procesamiento de la información). El factor "nivel de procesamiento" apareció como marginalmente significativo. Y los factores de "importancia de la comunicación y difusión de la información" y "nivel de comunicación" aparecieron sin diferencias significativas, es decir, todo el estudiantado les da la misma importancia con independencia del curso en el que estén. Atendiendo a las medias por curso podemos observar un patrón curioso donde el estudiantado de doctorado y máster es en todos los casos el que más importancia concede a las competencias y el que se siente mejor preparado en ellas. Sin embargo, es el estudiantado de menor curso (segundo) el que se suele sentir menos preparado y conceder menor importancia a las competencias, excepto en el factor de nivel adquirido de búsqueda de información donde sorprendentemente es el estudiantado de $5^{\circ}$ curso el que se siente menos preparado (ver Tabla VI).

\begin{tabular}{|l|r|r|c|}
\hline FACTOR & F & P & $\begin{array}{c}\text { ORDEN DE MAYOR A } \\
\text { MENOR }\end{array}$ \\
\hline Búsqueda importancia & 4.06 & .001 & $6^{\mathbf{o}}, 5^{\mathbf{o}}, 4^{\mathbf{o}}, 1^{\mathbf{o}}, 3^{\mathbf{o}}, 2^{\mathbf{o}}$. \\
\hline Búsqueda nivel & 5.45 & .000 & $6^{\mathbf{o}}, 1^{\mathbf{o}}, 2^{\mathbf{o}}, 4^{\mathbf{o}}, 3^{\mathbf{o}}, 5^{\mathbf{o}}$. \\
\hline Evaluación importancia & 5.05 & .000 & $6^{\mathbf{o}}, 5^{\mathbf{o}}, 4^{\mathbf{o}}, 1^{\mathbf{o}}, 3^{\mathbf{o}}, 2^{\mathbf{o}}$. \\
\hline Evaluación nivel & 8.46 & .000 & $6^{\mathbf{o}}, 1^{\mathbf{o}}, 4^{\mathbf{o}}, 3^{\mathbf{o}}, 5^{\mathbf{o}}, 2^{\mathbf{o}}$. \\
\hline Procesamiento importancia & 3.59 & .003 & $6^{\mathbf{o}}, 1^{\mathbf{o}}, 5^{\mathbf{o}}, 4^{\mathbf{o}}, 3^{\mathbf{o}}, 2^{\mathbf{o}}$. \\
\hline
\end{tabular}

Tabla VI. ANOVA para los 5 factores significativos en función del curso en el que están los participantes.

Realizamos un ANOVA para los hábitos de adquisición y los resultados fueron muy llamativos. Concretamente obtuvimos una F $(3,999)=291,42$ donde el estudiantado señalaba que adquiría las competencias principalmente de manera individual (media = 3.96 ), en segundo lugar en el aula (media $=1.87$ ), en tercer lugar en la biblioteca (media $=$ 1.33 ) y finalmente a través de cursos (media $=.7$ ). Todas las diferencias entre los distintos tipos de hábitos fueron significativas (.000). Se aprecia que los estudiantes señalan como fuente preferente de aprendizaje de las competencias informacionales el autoaprendizaje por medio de prácticas propias o de ayuda de compañeros/as.

\section{CONCLUSIONES}

Tomando como referencia los resultados del análisis cuantitativo observamos que el aprendizaje de las competencias informacionales es un tema relevante para los estudiantes de psicología. Sitúan en la cúspide de las competencias más importantes la de comunicación y difusión de la información, siendo esta conclusión congruente con los principios básicos de formación de estos profesionales. En el extremo opuesto, el estudiantado sitúa a dos competencias como menos importantes, la de búsqueda y 
tratamiento de la información, que curiosamente son las más próximas a competencias tecnológicas. Al mismo tiempo se observa coherencia entre las competencias que consideran más importantes y las que tienen mejor adquiridas a la vez que aquellas que consideran menos importantes se perciben como peor formados. Concretamente esto se ilustra con la observación del factor evaluación de la información.

Hay que concienciar al estudiante de la importancia que tienen estas competencias en su formación pues los resultados correlacionales desvelan que cuanta más importancia conceden a una competencia más autoeficaces se sienten en ella. Además al ser un resultado correlacional permite también la interpretación de que cuanto mayor autoeficacia logra el estudiante mayor importancia le concede a la competencia informacional. Esto implica que va a estar más motivado a su vez para aprender en esa competencia, lo que supondría una retroalimentación positiva en ese ciclo de mejora del aprendizaje. En este sentido debiera ir cualquier propuesta de intervención. Por ejemplo, la autoeficacia de los estudiantes podría ser potenciada si empleamos una amplia gama de actividades planificadas por niveles: conferencias, reuniones, cursos cortos, talleres, etc. Estos programas debieran ofrecer contenidos sobre competencias digitales para el acceso y organización de la información, especialmente en lo relacionado con fuentes electrónicas de información, estrategias de búsqueda de información, gestión de base de datos (Access, MySQL, etc.), gestores bibliográficos de referencia, programas estadísticos y hojas de cálculo y programas de ordenador.

En relación con los hábitos de aprendizaje detectamos que el estudiantado señala que adquiere mejor las competencias informacionales de manera individual. Este resultado es llamativo y tiene importantes implicaciones en el actual proceso de enseñanzaaprendizaje, ya que el aprendizaje individual no es sinónimo de aprendizaje autónomo. Para que el aprendizaje autónomo se produzca y éste sea significativo, entendemos que es necesario e imprescindible relacionar el aprendizaje individual con el aprendizaje guiado por el profesorado.

El estudiantado señala el aula como segundo hábito de aprendizaje de la competencia informacional, y esto consideramos que es una oportunidad para que el profesorado incluya contenidos y actividades relacionadas con el acceso y uso de la información como parte del currículum formativo. Este hecho cobra especial relevancia en el momento en el que señalan que los cursos como hábito de aprendizaje están en último lugar para adquirir las competencias, esto es, perciben que se forman mejor en competencia informacional en el aula que en cursos independientes.

Sin embargo la biblioteca ocupa la penúltima posición en la adquisición de las competencias informacionales. Este dato sorprende ya que aparece como una fuente menor pese a su potencial informativo para los futuros egresados que tendrán que manejar con frecuencia recursos y fuentes de información actualizadas. En general estas prácticas sobre hábitos de aprendizaje de la competencia informacional, implica que el estudiante de psicología posee un mejor autoconcepto de su capacidad formativa para adquirir las 
competencias informacionales sin la ayuda de la biblioteca. En general, se observa que dicen practicar el autoaprendizaje en todas aquellas competencias relacionadas con la evaluación y difusión de la información. Como conclusión sería aconsejable que tanto profesorado y bibliotecarios se sensibilizaran en que el acceso, uso, tratamiento y difusión de la información son vitales para el proceso formativo del estudiantado y la mejora de los resultados de aprendizaje. Esto implicaría abordar la formación en competencias informacionales desde una perspectiva integradora y en el marco de una planificación curricular adecuada. Una de las potencialidades de este estudio es ofrecer un diagnóstico desde la perspectiva del estudiantado a través de la autoevaluación de su competencia informacional. Somos conscientes de que es una perspectiva pionera en España, útil al aportarnos una primera radiografía que ha de ser contrastada con estudios de evaluación concretos que midan realmente lo que el estudiantado sabe hacer y no lo que cree o percibe que sabe hacer en contextos específicos. A partir de aquí sería conveniente diseñar propuestas de intervención encaminadas a mejorar las competencias de comunicación y difusión de la información ya que son las que más demandan y las de evaluación de la información porque son las que señalan como en las que están menos preparados.

\section{BIBLIOGRAFÍA}

ABBOTT, W. y PEACH, D. Building info-skills by degrees: embedding information literacy in university study. Paper presented at Virtual libraries: virtual communities: 21st IATUL Conference. 3-7 Jul 2000, Brisbane, Australia. [En linea] Disponible en: <http://www.iatul.org/doclibrary/public/Conf_Proceedings/2000/Abbott.rtf>.

[Consulta: 12 de diciembre de 2011].

ACRL. Information literacy competency standards for higher education. ACRL, 2000. [En linea] Disponible en: <http://www.ala.org/ala/acrl/acrlstandards/ informationliteracycompetency.htm>. [Consulta: 12 de diciembre de 2011].

ALA. Information Literacy Competency Standards for Higher Education. ALA, 1998. [En linea] Disponible en: <http://www.ala.org/ala/acrl/acrlstandards/standards.pdf >. [Consulta: 12 de diciembre de 2011].

ANECA. Titulo de Grado en Psicología. Madrid: ANECA, 2004.

APA (American Psychological Association). [En línea] Disponible en: <http:// www.apa.org/pubs/books/4313008.aspx>. [Consulta: 12 de abril de 2012].

BARTRAM, D. y ROE, R. Definition and assessment of competences in the context of the European Diploma in Psychology. European Psychologist, 2005, vol. 10, $\mathrm{n}^{\mathrm{o}}$ 2, p. 5-15.

BEHERENS, S.J. A Conceptual Analysis and Historical Overview of Information Literacy. College and Research Libraries, 1994, vol. 55, no 4, p. 309-22.

BLOOM, B.S. y KRATHWOHL, D.K. Taxonomy of Educational Objectives: the Classification of Educational Goals, by a committee of college and university examiners. Handbook I: Cognitive Domain. New York: Longmans, 1956.

BREVIK, P.S. y GEE, E.G. Information Literacy: Revolution in the Library. New York: Macmillan, 1989.

BRUCE, C. Seven Faces of Information Literacy. Adelaide: Auslib Press, 1997. 
CARTER, E.W. y DAUGHERTY, T.W. Library Instruction and Psychology. A Cooperative Effort. Technical Services Quarterly, 1998, vol. 16, nº 1, p. 33-41.

COLTHART, I.; BAGNALL, G.; EVANS, G.; et. al. The Effectiveness of SelfAssessment on the Identification of Learner Needs, Learner Activity, and Impact on Clinical Practice. Medical Teacher, 2008, no 30, p. 124-145.

DAUGHERTY, T.K. y CARTER, E.W. Assessment of Outcome-Focused Library Instruction in Psychology. Journal of Instructional Psychology, 1997, no 24, p. 29-33.

DOYLE, C.S. Information Literacy in an Information Society: a Concept for the Information Age. ERIC Digest ED 372763, 1994.

GONZÁLEZ, J. y WAGENAAR, (eds.). Tuning Educational Structures in Europe. Informe Final. Deusto: Universidad, 2003.

GROSS, M. y LATHAM, D. Undergraduate Perceptions of Information Literacy: Defining, Attaining, and Self-Assessing Skills. College \& Research Libraries, 2009, vol. 70, n 4, p. 336-350.

HAYES-BOHANAN, P. y SPIEVAK, E. You Can Lead Students to Sources, but Can You Make Them Think? College \& Undergraduate Libraries, 2008, vol. 15, $\mathrm{n}^{\circ}$ 1, $\mathrm{p}$. $173-210$.

HERNON, P.; DUGAN, R. y SHCWARTZ, C. (eds). Revisiting Outcomes Assessment in Higher Education. Westport, CT.: Libraries Unlimited, 2006.

HUSTON, M. Towards Information Literacy: Innovative Perspective for the 90 ' s. Library Trends, 1999, vol. 39, no 3, p. 12-17.

JULIEN, H. y BARKER, S. How High-School Students Find and Evaluate Scientific Information: A Basis for Information Literacy Skills Development. Library \& Information Science Research, 2009, vol 18, $\mathrm{n}^{\circ}$ 1, p. 1-6.

KUHLTHAU, C. Literacy and Learning in the Information Age. Proceedings of the International Conference on Information Literacy and Lifelong Learning, edited by Mei Mei Wu, National Normal University of Taiwan, May 1999.

LAMPERT, L. "Getting Psyched" About Information Literacy: A Successful FacultyLibrarian Collaboration for Educational Psychology and Counseling. The Reference Librarian, 2005, vol. 43, no 89, p. 5 -23.

LARKIN, J.E. y PINES, H.A. Developing Information Literacy and Research Skills in Introductory Psychology: A Case Study. Journal of Academic Librarianship, 2005, vol. $31, \mathrm{n}^{\circ} 1$, p. 40-45.

LIMBERG, L. y SUNDIN, O. (2006). Teaching information seeking: relating information literacy education to theories of information behaviour, Information Research, 2006, vol. $12, \mathrm{n}^{\mathrm{o}}$ 1. [En linea] Disponible en: <http://nformationR.net/ir/121/paper280.html>. [Consulta: 12 de diciembre de 2011].

MCKINNEY, P.; JONES, M. y TURKINGTON, S. Information literacy through inquiry A Level One Psycholy module at the University of Sheffield. Aslib Proceedings, 2011, vol. 63, nº 2/3, p. 221-240.

MAYBEE, C. Undergraduate Perceptions of Information Use: The Basis for Creating User-Centered Student Information Literacy Instruction. Journal of Academic Librarianship, 2006, vol. 32, nº 1, p. 79-85. 
MINISTERIO de Ciencia e Innovación. Real Decreto 1393/2007 de 29 de octubre, por el que se establece la ordenación de las enseñanzas universitarias oficiales. Madrid: BOE, 2007.

OECD. The definition and selection of key competencies. [en línea]. Executive Summary, 2005. [En linea] Disponible en: <http://www.pisa.oecd.org/dataoecd/47/61/ 35070367.pdf $>$. [Consulta: 12 de diciembre de 2011].

PAGLIA, A. y DONAHUE, A. Collaboration works: integrating information competencies into the psychology curricula. Reference Services Review, 2003, vol. 31, $\mathrm{n}^{\circ}$ 4, p. 320-328.

PAPALIA, D. y WENDKOS, S. Psicología. México: McGrawHill, 1993.

PINTO, M. (coord.). Portal ALFIN-EEES: Habilidades y competencies de gestión de información para aprender a aprender en el Marco del Espacio Europeo de Enseñanza Superior. Madrid: MEC, 2005. [En linea] Disponible en: <www.mariapinto.es/ alfineees>. [Consulta: 12 de diciembre de 2011].

PINTO, M. Design of the Il-Humass Survey on Information Literacy in Higher Education: A Self-Assessment Approach. Journal of Information Science, 2010, vol 36, $\mathrm{n}^{\mathrm{o}}$ 1, p. 86-103.

PINTO, M. An approach to the internal facet of Information Literacy using the ILHUMASS survey. Journal of Academic Library, 2011, vol. 37, no 2, p. 145-154.

RYCHEN, D. y SALGANIK, L. (eds.). Key Competencies for a Successful Life and a Well-Functioning Society. Göttingen: Hogrefe \& Huber, 2003.

SADLER, E. y GIVEN, L. Affordance theory: a framework for graduate students' information behavior. Journal of Documentation, 2007, vol. 63, $\mathrm{n}^{\circ}$ 1, p. 115-141.

SCONUL. Information Skills in Higher Education: A SCONUL Position Paper, 1999. [En linea] Disponible en: <http://www.sconul.ac.uk/groups/information_literacy/ seven_pillars.html>. [Consulta: 12 de diciembre de 2011].

SUTTON, E.D., et. al. Bibliographic Instruction in Psychology: A Review of the Literature. Reference Services Review, 1995, vol. 23, nº 3, p. 13-22.

THAXTON, L. Information Dissemination and Library Instruction in Psychology Revisited. Behavioral \& Social Sciences Librarian, 2002, vol. 21, nº 1, p. 1-14.

THAXTON, L.; FACCIOLI, M. y MOSBY, A. Leveraging collaboration for information literacy in psychology. Reference Services Review, 2004, vol. 32, nº 2, p. 185-189.

TUOMINEN, K.; SAVOLAINEN, R. y TALJA, S. Information Literacy as a Sociotechnical Practice. Library Quarterly, 2005, vol. 75, nº 3, p. 329-345.

WEBBER, S. y JOHNSTON, B. Working towards the information literate university. En G. Walton, A. Pope (Eds.) Information literacy: recognizing the need. Oxford: Chandos, 2006. 